\title{
Functions of two distinct Kupffer cells in the liver
}

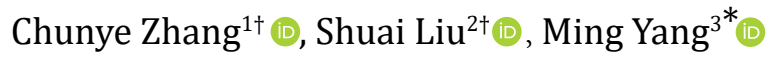 \\ ${ }^{1}$ Department of Veterinary Pathobiology, University of Missouri, Columbia, MO 65211, USA \\ ${ }^{2}$ The First Affiliated Hospital, Zhejiang University, Hangzhou 310006, Zhejiang, China \\ ${ }^{3}$ Department of Surgery, University of Missouri, Columbia, MO 65211, USA
}

${ }^{\dagger}$ The two authors contributed equally to the work.

*Correspondence: Ming Yang, Department of Surgery, University of Missouri, Columbia, MO 65211, USA. yangmin@health.missouri.edu

Academic Editor: Amedeo Lonardo, Azienda Ospedaliero-Universitaria di Modena, Italy

Received: October 7, 2021 Accepted: October 19, 2021 Published: December 31, 2021

Cite this article: Zhang C, Liu S, Yang M. Functions of two distinct Kupffer cells in the liver. Explor Med. 2021;2:511-5. https:// doi.org/10.37349/emed.2021.00067

\begin{abstract}
Tissue-resident macrophages play critically important roles in host homeostasis and pathogenesis of diseases, with the functions of phagocytosis, metabolism, and immune modulation. Recently, two research studies accomplished by a collaborated group of researchers showed that there are two populations of liver resident Kupffer cells (KCs), including a major cluster of differentiation 206 low expression (CD206 ${ }^{\text {low }}$ ) endothelial cell-selective adhesion molecule negative (ESAM) population (KC1) and a minor CD206 ${ }^{\text {high }} \mathrm{ESAM}^{+}$ population (KC2). Both KC1 and KC2 express KC markers, such as C-type lectin domain family 4 member $\mathrm{F}$ (CLEC4F) and T-cell membrane protein 4 (Tim4). In fatty liver, the frequency of KC2 was increased, and those KC2 expressed some markers like liver sinusoidal endothelial cells (LSECs), such as CD31 and ESAM. In addition, KC2 population had a relatively higher expression of CD36, as fatty acid transporter, which was implicated in the production of reactive oxygen species (ROS) and lipid peroxidation. Furthermore, this collaborated group also showed that KC2 can cross-present hepatocellular antigens to prime antiviral function of $\mathrm{CD}^{+} \mathrm{T}$ cells by sensing interleukin-2 (IL-2) in hepatitis B virus (HBV) replication-competent transgenic mice. Increasing evidence shows that targeting hepatic macrophages can prevent and reverse non-alcoholic fatty liver disease (NAFLD), with a new suggested name metabolic dysfunction-associated fatty liver disease (MAFLD) to include metabolic dysfunction-associated fatty liver diseases, such as viruses and alcohol. In summary, differentiating specific populations of hepatic macrophages is critically important for the treatment of MAFLD or NAFLD, and their overlaps. Markers specifically expressed on sub-types of hepatic macrophages may be applied for liver disease diagnosis.
\end{abstract}

\section{Keywords}

Macrophage, Kupffer cell, heterogeneity, chronic liver disease, cytokine, treatment

Macrophages play important roles in host homeostasis and pathogenesis of diseases, such as clearance of cellular debris and pathogens [1], metabolic function [2], and immune response [3]. Kupffer cells (KCs)

(C) The Author(s) 2021. This is an Open Access article licensed under a Creative Commons Attribution 4.0 International License (https://creativecommons.org/licenses/by/4.0/), which permits unrestricted use, sharing, adaptation, distribution and reproduction in any medium or format, for any purpose, even commercially, as long as you give appropriate credit to the original author(s) and the source, provide a link to the Creative Commons license, and indicate if changes were made. 
are predominant macrophages in healthy liver, which can self-renew or proliferate [4]. KCs reside along with liver sinusoidal endothelial cells (LSECs) and protect liver infection and injury with functions of phagocytosis, regulation of immunological tolerance, and metabolic function [5]. However, in pathogenic conditions, monocyte-derived macrophages from bone marrow can be recruited into the liver and be differentiated into macrophages and KC-like macrophages [6]. For example, In the development of metabolic dysfunction-associated fatty liver disease (MAFLD), liver resident KCs are lost and replaced by C-type lectin domain family 4 member F negative (CLEC4F-) monocyte-derived macrophages expressing osteopontin [7]. Monocyte-derived macrophages express high levels of lymphocyte antigen 6 complex (Ly6c) and C-C motif chemokine receptor 2 (CCR2). In addition, there is a very small population of macrophages different from KCs and monocytes-derived macrophages, including capsular macrophages and peritoneal macrophages in the liver [8].

Current studies using the single-cell RNA-sequencing (scRNA-seq) technique have revealed the presence of multiple macrophage subsets with distinct functions in various tissues [9]. Recently, Blériot et al. [10] reported that there are two phenotypically and functionally distinct populations of liver resident KCs in healthy mice, a major cluster of differentiation 206 low expression (CD206 ${ }^{\text {low}}$ )endothelial cell-selective adhesion molecule negative (ESAM) population (KC1) and a minor CD206 ${ }^{\text {high }} \mathrm{ESAM}^{+}$population (KC2). Both populations express liver resident macrophage markers such as adhesion $\mathrm{G}$ protein-coupled receptor E1 (Adgre1) encoding F4/80, integrin subunit alpha M (ITGAM) encoding CD11b, T-cell membrane protein 4 (Tim4), Clec4f, and colony-stimulating factor 1 receptor (Csf1r) (Figure 1). In contrast, KC2 expressed markers like LSECs, such as CD206, CD31 [platelet endothelial cell adhesion molecule 1 (Pecam1, also known as CD31)], lymphatic vessel endothelial hyaluronan receptor 1 (Lyve1), and ESAM. The frequency of KC2 was increased in the livers of mice fed a high-fat diet compared to that in mice fed a normal diet. The authors also found that functional depletion of KC2 or silencing fatty acid transporter CD36 resulting in a reduction of both reactive oxygen species (ROS) and the lipid peroxidation byproduct malondialdehyde (MDA), showing that KC2 functions on hepatic lipid peroxidation and oxidative stress [10].
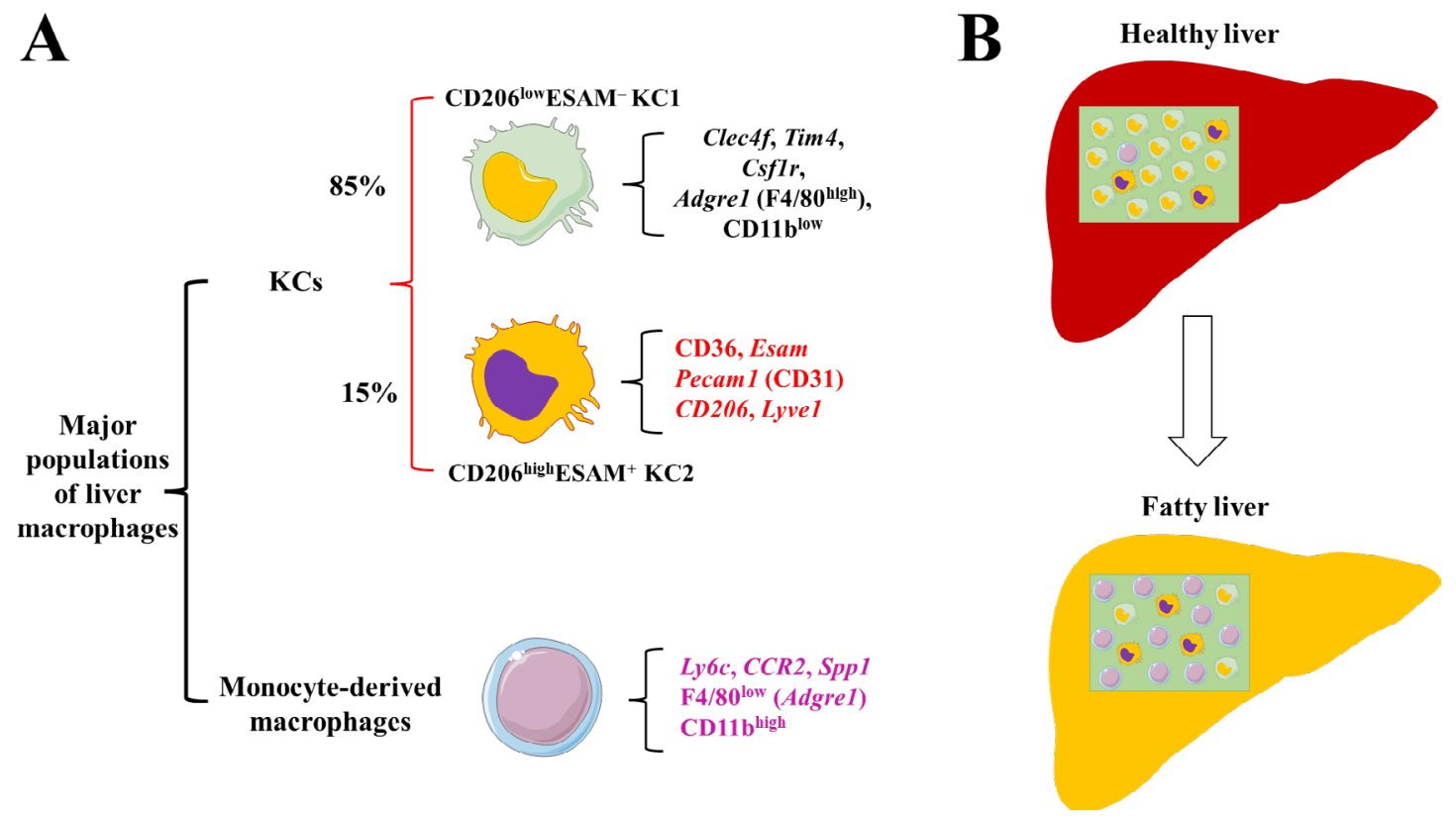

Figure 1. Macrophage populations in the healthy and fatty liver. (A) In healthy conditions, KCs are the most macrophages in the liver, including a major CD206 ${ }^{\text {low }} \mathrm{ESAM}^{-}$population $\left(\mathrm{KC} 1, \sim 85 \%\right.$ of $\mathrm{KCs}$ ) and a minor CD206 ${ }^{\text {high }} \mathrm{ESAM}^{+}$population (KC2, $\sim 15 \%$ of KCs). Both KC1 and KC2 express genes such as Clec4f, Tim4, Csf1r, Adgre1 (F4/80, high expression), and CD11b (low expression). In addition, KC2 expresses more LSEC-like genes including Esam, Pecam1, CD206, and Lyve1; (B) in fatty liver, the frequency of KC2 will be increased with increased expression of CD36, accompanied by an increase of monocyte-derived macrophages expressing genes including Ly6c, CCR2, secreted phosphoprotein 1 (Spp1), Adgre1 (F4/80, low expression), and $C D 11 \mathrm{~b}$ (high expression). The expressing levels of $\mathrm{F} 4 / 80$ and $C D 11 \mathrm{~b}$ are compared between KCs and monocyte-derived macrophages. Alteration of macrophage population will change hepatic inflammation, lipid metabolism, and fibrosis. Without effective prevention and treatment options, progression of MAFLD and liver fibrosis can result in liver cancer 
ESAM belongs to the immunoglobulin superfamily, which is expressed in vascular endothelial cells and plays pivotal roles in angiogenesis and cell migration [11]. ESAM is also expressed in dendritic cells (DCs), which is associated with the expression of another immunoglobulin superfamily protein signal regulatory protein $\alpha$ (SIRP $\alpha$ ) [12]. In addition, ESAM expression in hematopoietic progenitors plays a critical role in the erythroid recovery after a bone marrow injury [13].

CD206, the macrophage mannose receptor, is highly expressed in the alternatively activated macrophages (anti-inflammatory phenotype) [9]. In addition, CD206 is also expressed by DCs and LSECs in the liver, playing an essential role in endocytosis and phagocytosis [14]. Modulating a conformational change of CD206 by a synthetic amphipathic peptide sequence: KFRKAFKRFF (RP-182) can drive alternatively activated macrophages (M2-like) tumor-associated macrophages (TAMs) to an antitumor classically activated macrophages (M1-like) phenotype to enhance innate anti-tumor immunity [15]. However, the accumulation of $\mathrm{CD}_{206^{+}}$macrophages expressing tumor necrosis factor- $\alpha$ (TNF- $\alpha$ ) and granulocyte-macrophage colony-stimulating factor (GM-CSF) was shown in human fibrotic liver, and these macrophages were differentiated from monocytes stimulated by GM-CSF [16]. In vitro study further illustrated that lipopolysaccharide (LPS) induced monocytes conversion to $\mathrm{CD}_{206^{+}}$macrophage-like cells, which caused an increase in production of TNF- $\alpha$ that contributes to chronic inflammation [16].

Macrophage is one of the antigen-presentation cells, including KCs [17]. Collaborating with Blériot and co-workers, De Simone et al. [18] also found that CD45 $44 / 80^{+} \mathrm{CD} 11^{\text {bint }} \mathrm{TIM}-4^{+} \mathrm{KC}$ consist of CD206-ESAM-KC1 (about 70-85\%) and CD206 ${ }^{+} \mathrm{ESAM}^{+} \mathrm{KC} 2$ (about 15-30\%), another commentary paper. Specifically, KC2 can cross-present hepatocellular antigens to prime antiviral function of $\mathrm{CD}^{+} \mathrm{T}$ cells by sensing interleukin-2 (IL-2) in hepatitis B virus (HBV) replication-competent transgenic mice [18]. In addition, depletion of KC2 abrogated the function of IL- 2 to revert the dysfunction of T cells.

Macrophages play pivotal roles in liver homeostasis in physiological conditions and chronic liver diseases, including non-alcoholic fatty liver disease (NAFLD), MAFLD, liver fibrosis, and end-stage hepatocellular carcinoma (HCC) $[19,20]$. The major and significant difference between MAFLD and NAFLD is that NAFLD excludes some metabolic abnormalities with other etiologies that cause liver diseases such as alcohol-associated and viral-associated liver diseases, while MAFLD does not [21,22]. For example, inhibiting the infiltration of $\mathrm{CD}_{206}{ }^{+}$macrophages in the liver treated with an anti-GM-CSF neutralizing antibody can ameliorate liver fibrosis in HBV-infected humanized mice [16]. In addition, inhibiting the accumulation of monocyte-derived macrophages [e.g., blocking C-C motif chemokine ligand 2 (CCL2)/CCR2] signaling pathway can prevent liver inflammation, NAFLD, and liver fibrosis [23, 24]. Furthermore, macrophage markers such as soluble CD163 (sCD163) that is associated with liver inflammation and glucose metabolism in NAFLD, can be applied to assist diagnosis [25].

In summary, differentiating specific populations of hepatic macrophages is critically important for NAFLD and/or MAFLD treatments and their distinct expressing markers may be applied for liver disease diagnosis.

\section{Abbreviations}

Adgre1: adhesion G protein-coupled receptor E1

CCR2: C-C motif chemokine receptor 2

CD206 ${ }^{\text {low: }}$ cluster of differentiation 206 low expression

CLEC4F: C-type lectin domain family 4 member F negative

ESAM: endothelial cell-selective adhesion molecule negative

GM-CSF: granulocyte-macrophage colony-stimulating factor

KCs: Kupffer cells

LSECs: liver sinusoidal endothelial cells 
MAFLD: metabolic dysfunction-associated fatty liver disease

NAFLD: non-alcoholic fatty liver disease

\section{Declarations}

\section{Author contributions}

CZ, SL, and MY contributed conception and design of the study; CZ and SL wrote the first draft of the manuscript; all authors contributed to manuscript revision, read and approved the submitted version.

\section{Conflicts of interest}

The authors declare that they have no conflicts of interest.

\section{Ethical approval}

Not applicable.

\section{Consent to participate}

Not applicable.

\section{Consent to publication}

Not applicable.

Availability of data and materials

Not applicable.

\section{Funding}

Not applicable.

\section{Copyright}

(C) The Author(s) 2021.

\section{References}

1. Kim SH, Saeidi S, Zhong X, Gwak SY, Muna IA, Park SA, et al. Breast cancer cell debris diminishes therapeutic efficacy through heme oxygenase-1-mediated inactivation of M1-like tumor-associated macrophages. Neoplasia. 2020;22:606-16.

2. Yao J, Wu D, Zhang C, Yan T, Zhao Y, Shen H, et al. Macrophage IRX3 promotes diet-induced obesity and metabolic inflammation. Nat Immunol. 2021;22:1268-79.

3. Yu J, Green MD, Li S, Sun Y, Journey SN, Choi JE, et al. Liver metastasis restrains immunotherapy efficacy via macrophage-mediated T cell elimination. Nat Med. 2021;27:152-64.

4. Scott CL, Zheng F, De Baetselier P, Martens L, Saeys Y, De Prijck S, et al. Bone marrow-derived monocytes give rise to self-renewing and fully differentiated Kupffer cells. Nat Commun. 2016;7:10321.

5. Wen Y, Lambrecht J, Ju C, Tacke F. Hepatic macrophages in liver homeostasis and diseases-diversity, plasticity and therapeutic opportunities. Cell Mol Immunol. 2021;18:45-56.

6. Yang M, Kimchi ET, Staveley-O'Carroll KF, Li G. Astaxanthin prevents diet-induced NASH progression by shaping intrahepatic immunity. Int J Mol Sci. 2021;22:11037.

7. Remmerie A, Martens L, Thoné T, Castoldi A, Seurinck R, Pavie B, et al. Osteopontin expression identifies a subset of recruited macrophages distinct from Kupffer cells in the fatty liver. Immunity. 2020;53:641-57.e14.

8. Blériot C, Ginhoux F. Understanding the heterogeneity of resident liver macrophages. Front Immunol. 2019;10:2694. 
9. Zhang C, Yang M, Ericsson AC. Function of macrophages in disease: current understanding on molecular mechanisms. Front Immunol. 2021;12:620510.

10. Blériot C, Barreby E, Dunsmore G, Ballaire R, Chakarov S, Ficht X, et al. A subset of Kupffer cells regulates metabolism through the expression of CD36. Immunity. 2021;54:2101-16.e6.

11. Inoue M, Ishida T, Yasuda T, Toh R, Hara T, Cangara HM, et al. Endothelial cell-selective adhesion molecule modulates atherosclerosis through plaque angiogenesis and monocyte-endothelial interaction. Microvasc Res. 2010;80:179-87.

12. Washio K, Kotani T, Saito Y, Respatika D, Murata Y, Kaneko Y, et al. Dendritic cell SIRP $\alpha$ regulates homeostasis of dendritic cells in lymphoid organs. Genes Cells. 2015;20:451-63.

13. Sudo T, Yokota T, Okuzaki D, Ueda T, Ichii M, Ishibashi T, et al. Endothelial cell-selective adhesion molecule expression in hematopoietic stem/progenitor cells is essential for erythropoiesis recovery after bone marrow injury. PLoS One. 2016;11:e0154189.

14. Azad AK, Rajaram MV, Schlesinger LS. Exploitation of the macrophage mannose receptor (CD206) in infectious disease diagnostics and therapeutics. J Cytol Mol Biol. 2014;1:1000003.

15. Jaynes JM, Sable R, Ronzetti M, Bautista W, Knotts Z, Abisoye-Ogunniyan A, et al. Mannose receptor (CD206) activation in tumor-associated macrophages enhances adaptive and innate antitumor immune responses. Sci Transl Med. 2020;12:eaax6337.

16. Tan-Garcia A, Lai F, Sheng Yeong JP, Irac SE, Ng PY, Msallam R, et al. Liver fibrosis and CD206 ${ }^{+}$ macrophage accumulation are suppressed by anti-GM-CSF therapy. JHEP Rep. 2019;2:100062.

17. Bénéchet AP, De Simone G, Di Lucia P, Cilenti F, Barbiera G, Le Bert N, et al. Dynamics and genomic landscape of CD8 ${ }^{+} \mathrm{T}$ cells undergoing hepatic priming. Nature. 2019;574:200-5.

18. De Simone G, Andreata F, Bleriot C, Fumagalli V, Laura C, Garcia-Manteiga JM, et al. Identification of a Kupffer cell subset capable of reverting the $\mathrm{T}$ cell dysfunction induced by hepatocellular priming. Immunity. 2021;54:2089-100.e8.

19. Fukushima $\mathrm{H}$, Kono $\mathrm{H}$, Hirayama $\mathrm{K}$, Akazawa $\mathrm{Y}$, Nakata $Y$, Wakana $\mathrm{H}$, etal. Changes in function and dynamics in hepatic and splenic macrophages in non-alcoholic fatty liver disease. Clin Exp Gastroenterol. 2020;13:305-14.

20. Wu H, Zhong Z, Wang A, Yuan $\mathrm{C}$, Ning $\mathrm{K}$, Hu H, et al. LncRNA FTX represses the progression of non-alcoholic fatty liver disease to hepatocellular carcinoma via regulating the M1/M2 polarization of Kupffer cells. Cancer Cell Int. 2020;20:266.

21. Kang SH, Cho Y, Jeong SW, Kim SU, Lee JW; Korean NAFLD Study Group. From nonalcoholic fatty liver disease to metabolic-associated fatty liver disease: big wave or ripple? Clin Mol Hepatol. 2021;27:257-69.

22. Lin S, Huang J, Wang M, Kumar R, Liu Y, Liu S, et al. Comparison of MAFLD and NAFLD diagnostic criteria in real world. Liver Int. 2020;40:2082-9.

23. Baeck C, Wei X, Bartneck M, Fech V, Heymann F, Gassler N, et al. Pharmacological inhibition of the chemokine $\mathrm{C}-\mathrm{C}$ motif chemokine ligand 2 (monocyte chemoattractant protein 1) accelerates liver fibrosis regression by suppressing $\mathrm{Ly}-6 \mathrm{C}^{+}$macrophage infiltration in mice. Hepatology. 2014;59:1060-72.

24. Ehling J, Bartneck M, Wei X, Gremse F, Fech V, Möckel D, et al. CCL2-dependent infiltrating macrophages promote angiogenesis in progressive liver fibrosis. Gut. 2014;63:1960-71.

25. Rødgaard-Hansen S, St George A, Kazankov K, Bauman A, George J, Grønbæk H, et al. Effects of lifestyle intervention on soluble CD163, a macrophage activation marker, in patients with non-alcoholic fatty liver disease. Scand J Clin Lab Invest. 2017;77:498-504. 